\title{
An Easily Made, Low-Cost, Tissue-Like Ultrasound Phantom Material
}

\author{
Ronald O. Bude, MD, and Ronald S. Adler, MD, PhD
}

Ultrasound phantoms are generally of two types. One mimics the acoustic properties of tissue (with regard to the speed of sound, average attenuation, etc.). The main purpose of the other is to approximate the sonographic appearance of tissue. The latter is often used as a biopsy training aid. Those which mimic the acoustic properties of tissue have been constructed of agar with suspended graphite, ${ }^{1}$ polyurethane foam, ${ }^{2}$ and magnesium silicate gels, ${ }^{3}$ and are used chiefly as test phantoms for assessing diagnostic ultrasound imaging equipment or for studying the interaction of sound with tissue. They are generally not used for biopsy phantoms as they are either expensive or time-consuming to produce. Biopsy phantoms are simpler in construction, contain simulated cysts or masses, and are either echogenic or sonolucent. Echogenic media have consisted of a flour ${ }^{4}$ or cornstarch $^{5}$ in gelatin suspension, a silicium carbide powder in agar suspension, ${ }^{6}$ or agar. ${ }^{7}$ When properly made, their echogenicity simulates parenchymal tissue (except agar, which is only weakly echogenic and does not simulate parenchymal echo texture well); however, they can be laborious to produce, requiring stirring or rotation during cooling to insure that the scatterers or biopsy targets remain suspended and may require materials that are not widely available (agar, silicium carbide). Sonolucent media, on the other hand, consist of gelatin ${ }^{8}$ without suspended scatterers and are very easy and inexpensive to prepare. Unfortunately, they are also transparent (unless deeply colored), which allows the biopsy needle and biopsy target to be seen from the exterior. This does not mimic the in vivo situation, and makes the procedure artificially easy to perform. Additionally, the biopsy needle is artifi-

From the Department of Radiology, University of Michigan Medical School, Ann Arbor, Michigan. For reprints contact Ronald O. Bude, MD, Department of Radiology, University of Michigan Medical Center, Taubman Center 2910, 1500 E. Medical Center Drive, Ann Arbor, MI 48109-0326. cially easy to demonstrate sonographically in a sonolucent or hypoechoic medium compared to in vivo, as it stands out as a much more brightly echogenic structure in those media than in most tissues, again making the procedure artificially easy to perform. These shortcomings limit their usefulness in training for in vivo biopsy procedures.

We describe an easily made, low-cost preparation that possesses the advantages of both types of previously reported biopsy phantoms, with none of the disadvantages. It is visually opaque with an echogenicity which simulates parenchymal echo texture, yet is as easy and inexpensive to produce as clear gelatin phantoms.

\section{MATERIALS AND METHODS}

Ingredients in the following proportions, in the total volume desired, are stirred until completely dissolved, which takes only 1 or 2 minutes: 250 $\mathrm{mL}$ (approximately $1 \mathrm{cup}$ ) boiling water, $20 \mathrm{~g}$ of unflavored gelatin ( 3 packets of Knox brand gelatin), and $10 \mathrm{~g}$ (approximately 1 tablespoon) of sugar-free psyllium hydrophilic mucilloid fiber (brand name: sugar-free Metamucil). If the sugarcontaining variety is used, approximately three times the volume ( 3 tablespoons) will be required to obtain the same amount of psyllium fiber. To form a phantom without internal inclusions such as simulated "cysts" or "masses," this mixture is poured into the container selected (plastic bag, milk carton, etc.) and cooled until firm. One "unit volume" as described above, roughly cubic in shape, takes 1 hour to 2 hours to congeal in a refrigerator at $6^{\circ} \mathrm{C}$.

Forming the phantom in stages is the most satisfactory method to produce internal "cysts" or masses. A layer of mixture is poured into the container of choice and cooled until the surface is firm enough to support the intended inclusion, which is placed on it (the remaining mixture has 


\section{BUDE AND ADLER}

been partially submersed in a container of warm water to keep it from congealing). If the inclusion does not float, the remainder of the mixture is poured into the container and allowed to congeal. If the intended inclusion floats, a thin layer of mixture is poured into the container and the inclusion placed within this layer. The layer is allowed to congeal, trapping the inclusion. The remaining mixture is then poured over the trapped inclusion and cooled until firm.

"Cysts" are simulated with water-filled balloons, tips of examining gloves, ${ }^{4}$ grapes, ${ }^{8}$ or glycerine suppositories. "Masses" are simulated with carrot pieces, ${ }^{7}$ macaroni, ${ }^{7}$ olives, ${ }^{7}$ or hot dog pieces. If refrigerated, the phantom will not begin to undergo significant microbial degeneration for several weeks. We have found this mixture to be very "forgiving," and the gelatin concentration can be reduced by approximately $50 \%$ without a substantial change in firmness.

\section{DISCUSSION}

We have produced a tissue-like ultrasound phantom using a previously unreported material, psyl- lium hydrophilic mucilloid fiber, as the scattering medium (Figure 1). The echo texture of this material simulates thyroid or testicular parenchyma. The chief advantage that the use of this material provides, compared with previously reported tissue-like phantoms, ${ }^{4-7}$ is that after the mixture is initially prepared, further mixing is not required to maintain an even suspension of the scattering medium. This is in contrast to phantom materials using flour, cornstarch, or silicium carbide as the scattering medium, which require intermittent stirring during cooling until congealing of the mixture occurs, a process which can take more than 1 hour. Phantoms prepared using psyllium hydrophilic mucilloid fiber can be prepared much more easily, more quickly, and more reproducibly than phantoms made with previously reported materials. This material is very inexpensive, costing less than $\$ 1$ per unit volume (approximately $250 \mathrm{~mL}$ ), uses widely available materials, and is opaque (so that biopsy needles and targets can only be seen sonographically and not with the naked eye). A strip of gelatinimpregnated gauze, which simulates skin and

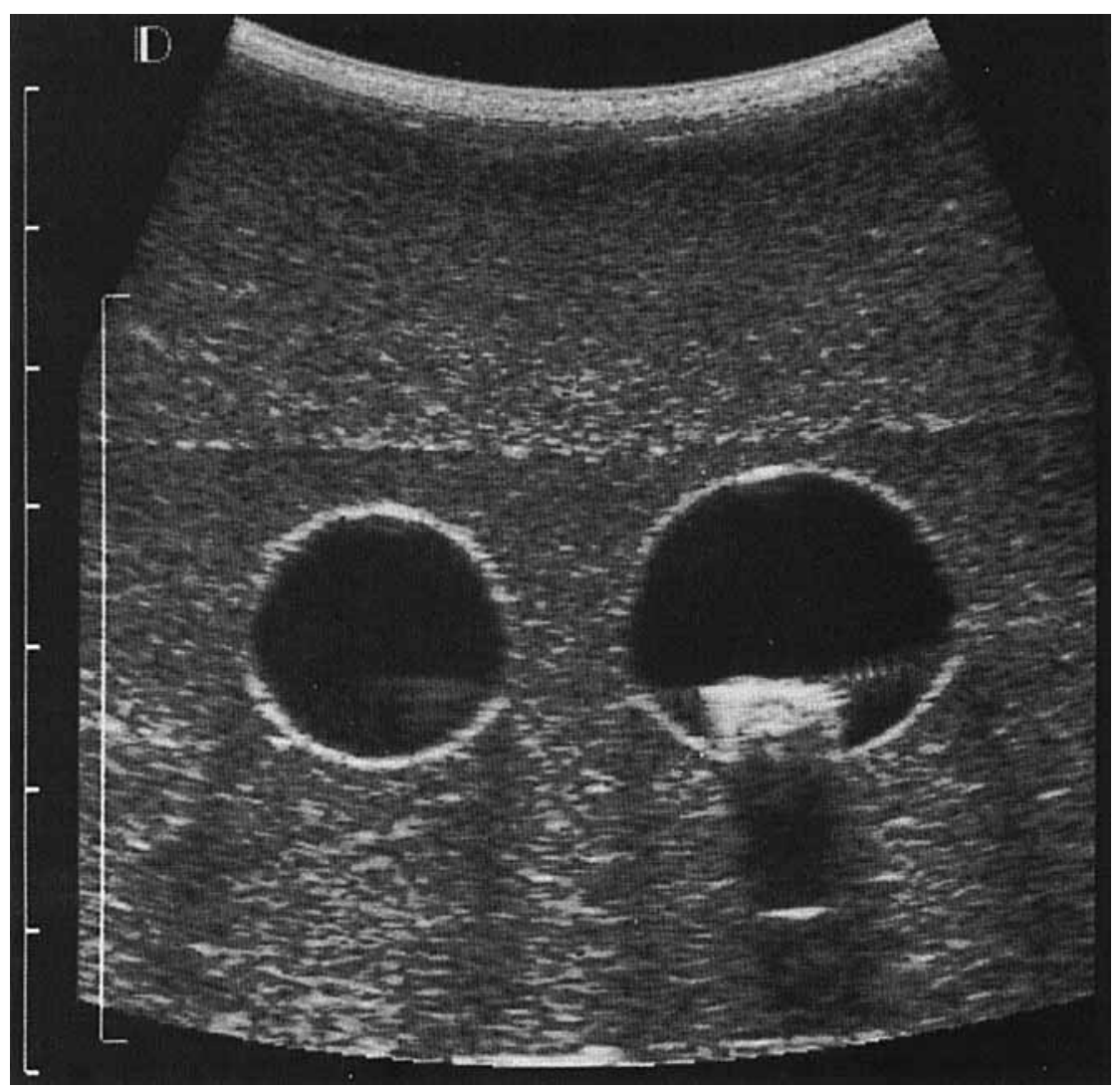

FIGURE 1. Image of a phantom obtained with a 5-MHz curvilinear array transducer (Diasonics Corp., Milpitas, CA). Note the homogeneous "parenchymal" echotexture. "Cysts" are water-filled balloons, with the larger one containing a small pebble. The echogenic line immediately superior to the "cysts" represents the boundary between successively poured layers. 
helps prevent surface laceration, can be coated onto the scanning surface with a small amount of gelatin to prolong the life of the phantom. ${ }^{6}$

\section{REFERENCES}

1. Burlew MM, Madsen EL, Zagzebski JA, et al: A new ultrasound tissue-equivalent material. Radiology 134:517, 1980.

2. Ophir J: Ultrasound phantom material. Br J Radiol 57:1161, 1984.

3. Sheppard J, Duck FA: Ultrasonic tissue-equivalent materials using inorganic gel mixtures. $\mathrm{Br} J$ Radiol 55:667, 1982.
4. McNamara MP, McNamara ME: Preparation of a homemade ultrasound biopsy phantom. $J$ Clin $\mathrm{Ul}$ trasound 17:456, 1989.

5. Rubin JM, Adler RS, Bude RO, et al: Clean and dirty shadowing at US: a reappraisal. Radiology 181:231, 1991.

6. Fredfeldt KE: An easily made ultrasound biopsy phantom. J Ultrasound Med 5:295, 1986.

7. Silver B, Metzger TS, Matalon TA: A simple phantom for learning needle placement for sonographically guided biopsy. AJR 154:847, 1990.

8. Fornage B: A simple phantom for training in ultrasound-guided needle biopsy using the freehand technique. J Ultrasound Med 8:701, 1989. 